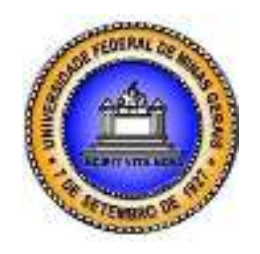

\title{
IDENTIFICAÇÃO DOS NÍVEIS DE BURNOUT EM UM HOSPITAL PÚBLICO E APLICAÇÃO DA ESCALA MBI-GS ${ }^{1}$
}

\section{IDENTIFICATION OF THE LEVELS OF BURNOUT IN A PUBLIC HOSPITAL AND APPLICATION OF SCALE MBI-GS}

\section{IDENTIFICACIÓN DE LOS NIVELES DE BURNOUT EN UN HOSPITAL PÚBLICO Y LA APLICACIÓN DE LA ESCALA MBI-GS}

\author{
MARCELO DA SILVA SCHUSTER \\ Universidade Federal de Santa Maria \\ marcelo.schuster@gmail.com
}

VALÉRIA DA VEIGA DIAS

Universidade Federal do Rio Grande do Sul

valeria-adm@hotmail.com

\author{
LUCIANA FLORES BATTISTELLA \\ Universidade Federal de Santa Maria Brasil \\ $\underline{\text { lutti@ufsm.br }}$
}

\section{MÁRCIA ZAMPIERI GROHMANN}

Universidade Federal de Santa Maria Brasil

marciazg@gmail.com

\section{RESUMO:}

O presente estudo foi desenvolvido em um hospital público objetivando a mensuração dos níveis de Burnout para os profissionais de diversos cargos. A metodologia utilizada foi quantitativa, com uso da coleta de dados com instrumento estruturado, a escala MBI-GS. Foram coletadas 173 respostas e realizados limpeza da escala e verificação da fatorabilidade, testes de confiabilidade, testes de hipótese. Como resultados identificou-se adequação da escala e boa fatorabilidade, a confiabilidade da MBI-GS foi ótima, com um Alfa de Cronbach de 0,87. Verificou-se níveis moderados de Burnout $(1,52)$ para os fatores Exaustão Emocional $(2,50)$ e Cinismo $(1,05)$ e nível alto para Eficácia no Trabalho $(5,0)$. Quanto aos testes de hipóteses foi confirmado que a síndrome de Burnout se desenvolve independente de qual cargo ou área a pessoa trabalha, também foi reforçado que as mulheres estão mais predispostas a apresentarem níveis mais altos de Burnout que os homens. Como limitantes de análise evidencia-se que apesar da aplicabilidade e respaldo internacional da escala, foram encontrados poucos estudos que utilizaram a mesma, não sendo possivel realizar a comparação dos indicadores encontrados. PALAVRAS-CHAVE: Burnout, Hospital Público, Servidores, Exaustão.

\begin{abstract}
:
This study was conducted in a public hospital aiming to measure levels of burnout for professionals in various positions. The methodology used was quantitative, using the data collection with structured instrument, the MBI-GS scale. 173 responses were collected and made cleaning the scale and check factorability, reliability testing, hypothesis testing. The results identified the adequate range and good factorability, the reliability of the MBI-GS was excellent, with a Cronbach's alpha of 0.87. There was moderate levels of burnout (1.52) for the
\end{abstract}

\footnotetext{
${ }^{1}$ Submetido em 23 de junho de 2013. Aceito em 10 de outubro de 2013. O artigo foi avaliado segundo o processo de duplo anonimato e avaliado pelo editor. Editores responsáveis: Márcio Augusto Gonçalves e Lucas Maia dos Santos. Reprodução parcial ou total e trabalhos derivativos permitidos com a citação apropriada da fonte.
} 

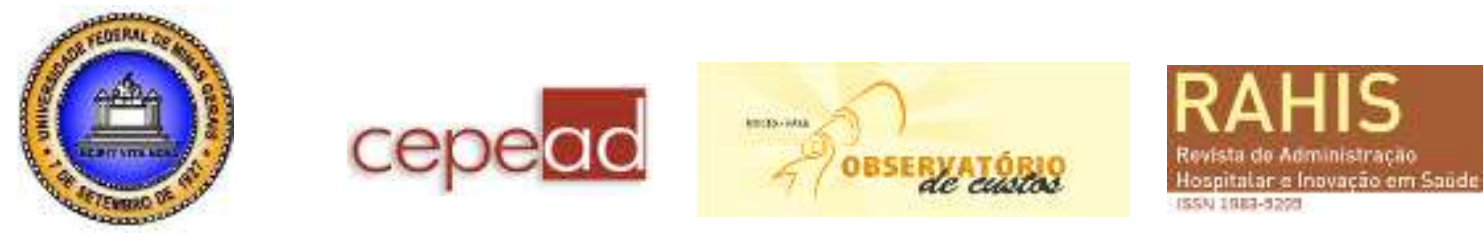

factors Emotional Exhaustion (2.50) and cynicism (1.05) and high level for Workplace Effectiveness (5.0). As for hypothesis testing confirmed that the burnout syndrome develops regardless of which position or area the person works, was also reinforced that women are more prone to present higher levels of burnout than men. As limiting the analysis shows that despite the applicability of the scale and international backing, found few studies that used the same, it is not possible to make a comparison of the indicators found.

KEYWORDS: Burnout, Public Hospital, Servers, Exhaustion.

\section{RESUMEN}

Este estudio fue realizado en un hospital público con el objetivo de medir los niveles de burnout para profesionales en varias posiciones. La metodología utilizada fue cuantitativa, mediante la recopilación de datos con el instrumento estructurado, la escala MBI-GS. 173 respuestas se recogieron y llevaron a cabo la limpieza y verificación de escala factorabilidad, pruebas de fiabilidad, pruebas de hipótesis. Como resultado se identificaron idoneidad de escala y buena factorabilidad, la fiabilidad de la MBI-GS fue excelente, con un alfa de Cronbach de 0,87 . Hubo niveles moderados de burnout (1.52) para el agotamiento emocional $(2,50)$ y el cinismo $(1,05)$ y de alto nivel de los factores de Efectividad en el Trabajo (5.0). En cuanto a la prueba de hipótesis confirmó que el síndrome de Burnout se desarrolla independientemente de la posición o área trabaja la persona, también se reforzó que las mujeres tienen más probabilidades de presentar niveles altos de burnout que los hombres. Como limitar el análisis es evidente que a pesar de la aplicabilidad de la escala y el respaldo internacional, pocos estudios utilizando el mismo, no siendo posible hacer una comparación de los indicadores encontrados fueron encontrados.

PALABRAS CLAVE: Burnout, Hospital Público, Servidores, Agotamiento.

\section{INTRODUÇÃO}

O ambiente hospitalar é um local onde os trabalhadores permanecem e dedicam à maior parte de seu dia, liberando suas potencialidades e compartilhando a meta coletiva de ajudar as pessoas (BACKES; LUNARDI FILHO; LUNARDI, 2006). Porém, por trabalhar com questões cruciais da vida do ser humano esse ambiente pode impactar significativamente a vida dos trabalhadores, influenciando o comportamento e os objetivos pessoais de cada profissional e afetando diretamente o modo de prestar assistência ao paciente.

A forma como se estrutura este tipo de organização aliada à dinâmica de funcionamento dos processos, rotinas de trabalho, interação das pessoas e adequação as mudanças do ambiente podem gerar tensões ao longo do período de trabalho, que muitas vezes os trabalhadores não conseguem aliviar em seu período de descanso. Essa tensão psicológica somadas às tensões crônicas do dia-a-dia e ao estado de exaustão física, emocional e mental existente em um ambiente de trabalho com essas características foi definida por Etzion (1984) como Síndrome de Burnout.

Maslach (2003) definiu Burnout como uma resposta à prolongada exposição aos estressores crônicos emocionais e interpessoais no trabalho, que despertam no trabalhador uma sensação de fadiga e tensão e ainda um sentimento de estar sofrendo uma sobrecarrega no trabalho. Essa condição pode despertar no indivíduo a necessidade de criar estratégias para 

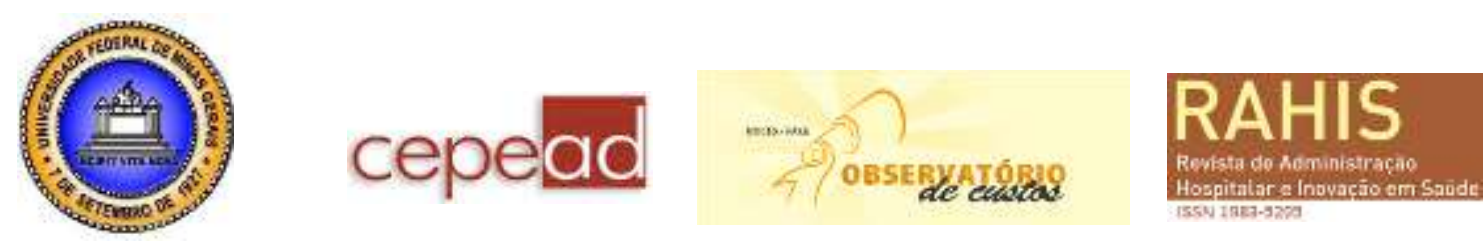

escapar dessa tensão através da fuga dos propósitos da sua função, que gera uma ineficiência no ambiente de trabalho.

Maslach e Goldberg (1998) afirmam ainda que o Burnout é um risco ocupacional para várias pessoas orientadas para profissões, tais como serviços humanos, educação e cuidados de saúde, que podem resultar nos sentimentos de exaustão avassaladora; sentimentos de frustração, raiva e cinismo, e uma sensação de ineficácia e fracasso.

O ambiente hospitalar, escolhido como objeto deste estudo, objetiva em todas as suas ações as relações de cuidados e preservação com a vida, estruturado em serviços de diversos profissionais de saúde (médicos, enfermagem, nutrição, fisioterapia) e de staff (administrativos, agendamentos, recepção, levantamento de fundo) e a preocupação com a manutenção de um bom ambiente de trabalho aumenta, isso porque os reflexos de altos níveis de Burnout podem implicar em consequências diretas para a vida das pessoas envolvidas.

Sendo assim, o presente estudo foi desenvolvido em um hospital público objetivando a mensuração dos níveis de Burnout para os profissionais ocupantes de cargos diversos. A metodologia identificada mais adequada para esta investigação foi de natureza quantitativa, com uso da coleta de dados com instrumento estruturado, a escala MBI-GS, desenvolvida para aplicação junto á uma população não segmentada ou especificada. A estrutura do trabalho é composta desta introdução, conceitos e desenvolvimento do Burnout, metodologia utilizada, apresentação dos resultados e considerações finais.

\section{BURNOUT}

O termo Burnout foi cunhado por Herbert Freudenberger em 1974, começando a aparecer com alguma regularidade na década de 1970 nos Estados Unidos, especialmente entre as pessoas que trabalham nos serviços humanos (MASLACH; SCHAUFELI; LEITER, 2001).

Burnout é caracterizado como uma síndrome, inicialmente estudada em cuidadores, por exemplo, os empregados de serviços sociais, enfermeiros ou funcionários do hospital, (BEER, 1992 apud PRUESSNER; HELLHAMMER; KIRSCHBAUM, 1999). Maslach et al. (2001) descrevem como uma síndrome psicológica em resposta a uma prolongada exposição a estressores crônicos emocionais e interpessoais no trabalho e a constitui por três dimensões, exaustão, cinismo e ineficácia.

Corroborando Schaufeli e Greenglass (2001) definem as dimensões de Burnout como sentimento de estar emocionalmente sobrecarregados e drenado por outros (exaustão emocional); resposta indiferente a realização da tarefas do trabalho, muitas vezes caracterizado pela fuga (cinismo); e declínio nos sentimentos um do de competência e realização bem sucedida em seu trabalho com as pessoas (Eficácia no trabalho).

Ahola et al. (2006) explicam que a exaustão emocional refere-se a sentimentos de excesso de esforço, cansaço ou fadiga resultante de envolvimento a longo prazo em uma situação de excesso de trabalho exigente. Jackson, Schwab e Schuler (1986) corroboram afirmando que a Exaustão presume estados anteriores de alta excitação, em contraste com o tédio, o que pode ser experimentado por seu detentor, cujo trabalho é monótono ou chato, a exaustão é mais propicia a trabalhadores cujo trabalho é muito envolvente.

A segunda dimensão do Burnout é o cinismo refere-se a distanciar-se do próprio trabalho e o desenvolvimento de atitudes negativas em relação ao trabalho em geral. (BAKKER; DEMEROUTI; SCHAUFELI, 2002). Ahola et al. (2006) e Jackson et al. (1986) corroboram afirmando que o cinismo reduz a energia disponível para a realização do trabalho e para o 

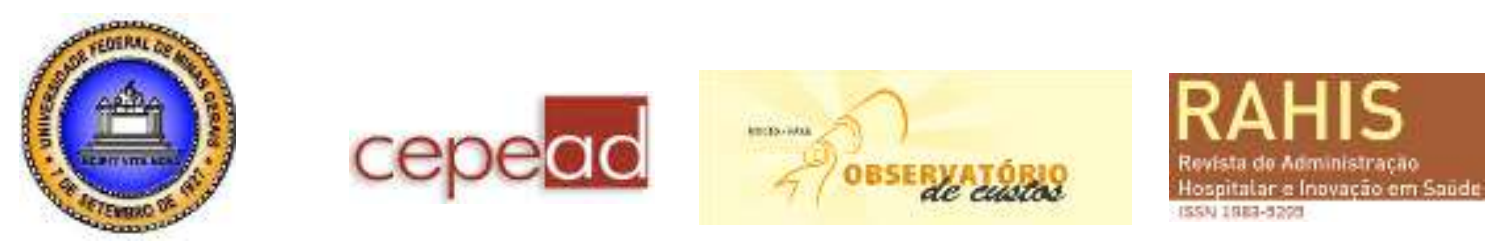

desenvolvimento de soluções criativas para os problemas que apresenta trabalhos, e diminui o potencial do trabalho para a construção de eficácia profissional, refletindo em uma atitude de indiferente e distante em relação ao trabalho, a retirada do mesmo, e falta de entusiasmo.

A terceira dimensão de Burnout refere-se à crença de que o trabalhador não é mais eficaz no cumprimento das responsabilidades do trabalho (BAKKER; VAN EMMERIK; EUWEMA, 2006). Para Maslach et al. (2001) esta dimensão representa a auto-avaliação de Burnout referindo-se a sentimentos de incompetência e falta de realização e produtividade no trabalho.

Bakker et al. (2006) afirmam que indivíduos com Burnout sofrem com a sensação de fadiga comportando-se com indiferença para com o seu trabalho e os clientes e apresentando um desempenho reduzido. Lee e Ashforth (1996) propõem que isso acontece quando certos recursos valiosos são perdidos, são insuficientes para atender às demandas ou não produzir os retornos esperados.

Além disso, Pruessner et al. (1999) afirmam a síndrome tendem a apresentar dor inespecífica nos indivíduos, com sintomas físicos como cansaço, fadiga, dores de cabeça e perturbações do sono, reduzida capacidade de atenção, sentimentos de falta de sentido, apatia ou desinteresse do trabalho provenientes de exigências do trabalho que incluem a ambiguidade do papel, conflito de papéis, eventos estressantes, pesada carga de trabalho e pressão.

Schaufeli e Greenglass (2001) ratificam que as exigências feitas aos trabalhadores por indivíduos e a falta de tempo para cumprir as exigências tornam-se fontes de estresse, variando entre profissões e quando isso acontece os torna altamente vulneráveis ao Burnout. MonteroMarin et al. (2011) alegam que embora a síndrome de Burnout tende a ser mais prevalente em assistência ou serviço de cuidados humanos, tem sido observada em todos os tipos de ocupações. O que propõem a nossa primeira hipótese:

H1: Não existe diferença entre as profissões relacionadas à área médica e as relacionadas às áreas administrativas.

Quando mensurado o Burnout em relação ao gênero, Dahlin, Fjell e Runeson (2010) afirmam que as mulheres apresentam índices maiores de Burnout que os homens, apresentandose assim a segunda hipótese.

H2: Mulheres apresentam índices maiores de Burnout que os Homens;

Ahola et al. (2006) em sua pesquisa encontrou que a síndrome de Burnout aumenta com a idade, apresentando aumento no nível de o Burnout após cada 10 anos, em primeiro lugar depois de 41 anos de idade e, em seguida, novamente após 52 anos de idade. Surgindo a terceira hipótese deste estudo.

H3: A síndrome de Burnout aumenta proporcionalmente com a idade.

A quarta hipótese diz respeito ao tempo de serviço dos trabalhadores, propondo que quanto mais tempo na instituição maior tende a ser o nível de Burnout apresentado, conforme encontrou (AHOLA et al., 2006).

H4: Quanto maior o tempo de serviço na organização, maior o nível de Burnout apresentado.

Para a mensuração do Burnout será utilizada a escala Maslach Burnout Inventory (MBI) que segundo Halbesleben e Buckley (2004) é a medida dominante no Burnout. Por o ambiente ser caracterizado pela diversidade de cargos, será utilizada a versão para a população geral, a MBI-GS (LINDBLOM et al., 2006).

A MBI-GS mede relações dos entrevistados com seu trabalho em um continuum de envolvimento com Burnout, através de três dimensões, a exaustão emocional, o cinismo e a eficácia profissional (SCHAUFELI; GREENGLASS, 2001). 


\section{cepead}

\section{MÉTODO}

Este estudo caracteriza-se como uma pesquisa descritiva de natureza quantitativa, utilizando como instrumento de coleta de dados o Maslach Burnout Inventory - General Survey (MBI-GS) que é um instrumento utilizado para mensurar Burnout em praticamente qualquer contexto ocupacional trabalho (MASLACH; SCHAUFELI; LEITER, 2001).

A MBI-GS é composta de três dimensões, Exaustão Emocional (EE) com seis variáveis, Cinismo $(\mathrm{CI})$ com quatro variáveis e Eficácia no Trabalho (ET) com seis variáveis, composta de uma escala likert que varia de 0 a 6, variando de Nunca, Algumas vezes, ao ano ou menos, Uma vez por mês ou menos, Algumas vezes durante o mês, Uma vez por semana, Algumas vezes durante a semana, até, todo dia. As 16 variáveis da escala são demonstradas no Quadro 1 .

\begin{tabular}{|ll|}
\hline CÓD. & \\
EE1 & Sinto-me emocionalmente esgotado com o meu trabalho \\
EE2 & Sinto-me esgotado no final de um dia de trabalho \\
EE3 & Sinto-me cansado quando me levanto pela manhã e preciso encarar outro dia de trabalho \\
EE4 & Trabalhar o dia todo é realmente motivo de tensão para mim \\
EE5 & Sinto-me acabado por causa do meu trabalho \\
EE6 & Só desejo fazer meu trabalho e não ser incomodado \\
CI1 & Sou menos interessado no meu trabalho desde que assumi essa função \\
CI 2 & Sou menos entusiasmado com o meu trabalho \\
CI 3 & Sou mais descrente sobre a contribuição de meu trabalho para algo \\
CI 4 & Duvido da importância do meu trabalho \\
ET1 & Sinto-me entusiasmado quando realizo algo no meu trabalho \\
ET2 & Realizo muitas coisas valiosas no meu trabalho \\
ET3 & Posso efetivamente solucionar os problemas que surgem no meu trabalho. \\
ET4 & Sinto que estou dando uma contribuição efetiva para essa organização. \\
ET5 & Na minha opinião, sou bom no que faço \\
ET6 & No meu trabalho, me sinto confiante de que sou eficiente e capaz de fazer com que as coisas \\
\hline
\end{tabular}

Quadro 1 - Variáveis por fator de Burnout MBI-GS

Fonte: Ferreira (2011).

Na mensuração dos índices de Burnout com a escala MBI-GS, são definidos que valores até 1,33 são considerados baixos, entre 1,34 e 2,43 intermediários a acima de 2,43 altos, conforme determina Mclaurine (2008), o mesmo autor ainda especifica os índices por fatores, conforme Tabela 01 a seguir.

Tabela 01 - Amplitude de Valores para mensuração dos níveis de Burnout

\begin{tabular}{lccc}
\hline & Baixo & Moderado & Alto \\
Burnout & $<1,33$ & $1,34-2,43$ & $>2,43$ \\
Exaustão Emocional & $<2,0$ & $2,1-3,19$ & $>3,20$ \\
\hline
\end{tabular}



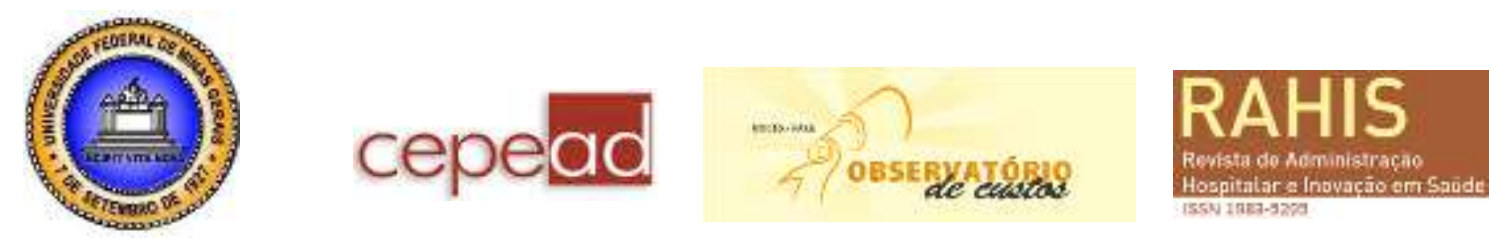

\begin{tabular}{lccc}
\hline Cinismo & $<1,0$ & $1,01-2,10$ & $>2,20$ \\
Eficácia no Trabalho & $>4,0$ & $4,01-4,99$ & $>5,0$ \\
\hline
\end{tabular}

Fonte: Mclaurine (2008)

A realização da coleta de dados deste estudo foi realizada no período de março a abril de 2013 em um Hospital Público que possui 1.265 servidores da cidade de Santa Maria, após a aprovação no comitê de ética da instituição.

O público alvo da pesquisa foram os servidores do hospital em questão, envolvendo participantes de diversos cargos que para facilitar a apresentação dos resultados foram divididos em duas categorias, cargos relacionados com a área médica (ex. médicos, enfermeiros, auxiliares de enfermagem, etc.) e cargos relacionados com a área administrativa (administrador, contado, assistentes administrativos, etc.).

A coleta de dados implicou na distribuição de 350 questionários nos diversos setores e realizada uma visita para recolher os questionários após uma semana, posteriormente após três dias e a última visita após quatro dias.

Foram obtidas 173 respostas (questionários) e os dados coletados foram tabulados por meio do Microsoft Excel e posteriormente, analisados estatisticamente utilizando o software SPSS. Para a identificação das variáveis mais importantes do modelo e das variáveis menos significativas foram realizados os testes do Coeficiente Alfa de Cronbach e Análise Fatorial. O Alfa de Cronbach (ou coeficiente alfa) é a medida comumente utilizada de confiabilidade para um conjunto de dois ou mais indicadores de construto. Os valores variam entre 0 e 1 com as medidas mais altas indicando maior confiabilidade entre os indicadores (Hair et al., 2009).

Após conhecer a estrutura fatorial da escala e sua confiabilidade no ambiente pesquisado foram analisadas as médias encontradas para o construto Burnout e suas dimensões, após procedeu-se os testes de hipóteses, conforme é apresentado no item seguinte.

\section{RESULTADOS}

A amostra deste estudo foi composta de 173 respondentes, sendo 30,6\% do sexo masculino e $69,4 \%$ do sexo feminino, dos participantes da pesquisa $61 \%$ exerce cargos ligados a saúde (ex. médico, enfermeiro etc.) e $39 \%$ cargos de cunho administrativo. Quanto à idade dos participantes $44,2 \%$ afirmam ter idade superior a 48 anos, demonstrando maiores experiências de vida, sendo que do total 33,3\% tem acima de 20 anos de serviço, 36,3\% tem entre 13 e 20 anos de serviço, 19,3\% entre 7 e 12 anos e $21,1 \%$ até 6 anos, o que caracteriza a população do estudo como experiente em suas funções.

Com a população de 173 questionários válidos realizou-se a análise fatorial para condensar as informações das diversas variáveis utilizadas em um número menor, representativa e com perdas mínimas de informações (HAIR et al., 2009). Foi adotado o método exploratório, com a rotação Varimax que para Hair et al. (2009) é o método considerado superior aos outros, por conseguir uma estrutura fatorial simplificada.

A utilização deste método de rotação fatorial teve o intuito de maximizar o peso de cada variável dentro de cada fator e como critério de extração foi definido valores da carga fatorial e comunalidade abaixo de 0,5 .

A escala utilizada apresenta em sua literatura três fatores, sendo eles exaustão emocional, Cinismo e Eficácia no Trabalho, após rodar os dados a escala apresentou um KaiserMeyer-Olkin (KMO) de 0, 837, o qual Pestana e Gageiro (2003) afirmam comparar as 


\section{cepead}
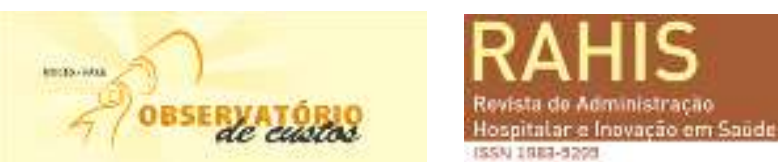

correlações de ordem zero com as correlações parciais observadas entre as variáveis, onde quanto mais próximo de 1 for, melhor é considerado. O teste de Barllett apresentou um Quiquadrado de 1302,723 com sig 0,000, mostrando que existe correlação entre as variáveis e sendo favorável.

O modelo, inicialmente, apresentou 4 fatores que explica uma variância de 70,08\%, apresentando uma variável (EE6) com Cargas Fatoriais abaixo de 0,5. Desta forma, optou-se por realizar a extração da ET6 com o intuito de obter variáveis com cargas fatoriais maiores que 0,5 .

Após a extração o modelo apresentou três fatores que explicam $65,72 \%$ da variância, com todas as variáveis com cargas e comunalidades acima de 0,5 e um KMO de 0,843 e um teste de Barllett com Qui-quadrado de 1262,696 e sig 0,000. Os fatores foram agrupados conforme a literatura, conforme pode ser conferido na Tabela 2.

Tabela 2 - Análise fatorial de Burnout

\begin{tabular}{|c|c|c|c|}
\hline \multirow{2}{*}{ Variáveis } & \multicolumn{3}{|c|}{ Fatores } \\
\hline & $\mathbf{E E}$ & EPT & $\mathbf{D P}$ \\
\hline EE2 - Sinto-me esgotado no final de um dia de trabalho & 0,87 & & \\
\hline EE1 - Sinto-me emocionalmente esgotado com o meu trabalho & 0,81 & & \\
\hline $\begin{array}{l}\text { EE3 - Sinto-me cansado quando me levanto pela manhã e preciso encarar } \\
\text { outro dia de trabalho }\end{array}$ & 0,77 & & \\
\hline EE4 - Trabalhar o dia todo é realmente motivo de tensão para mim & 0,77 & & \\
\hline EE5 - Sinto-me acabado por causa do meu trabalho & 0,74 & & \\
\hline ET5 - Na minha opinião, sou bom no que faço & & 0,79 & \\
\hline ET2 - Realizo muitas coisas valiosas no meu trabalho & & 0,79 & \\
\hline ET4 - Sinto que estou dando uma contribuição efetiva para essa organização. & & 0,77 & \\
\hline $\begin{array}{l}\text { ET6 - No meu trabalho, me sinto confiante de que sou eficiente e capaz de } \\
\text { fazer com que as coisas aconteçam. }\end{array}$ & & 0,76 & \\
\hline ET1 - Sinto-me entusiasmado quando realizo algo no meu trabalho & & 0,64 & \\
\hline $\begin{array}{l}\text { ET3 - Posso efetivamente solucionar os problemas que surgem no meu } \\
\text { trabalho. }\end{array}$ & & 0,62 & \\
\hline CI3 - Sou mais descrente sobre a contribuição de meu trabalho para algo & & & 0,84 \\
\hline CI 2 - Sou menos entusiasmado com o meu trabalho & & & 0,79 \\
\hline CI 4 - Duvido da importância do meu trabalho & & & 0,79 \\
\hline CI 1 - Sou menos interessado no meu trabalho desde que assumi essa função & & & 0,66 \\
\hline
\end{tabular}

Fonte: Dados da Pesquisa

Após a realização da análise fatorial procedeu à análise de confiabilidade, para mostrar até que ponto as escalas produzem resultados consistentes, livres de erros aleatórios (MALHOTRA, 2012).

Foi realizada a análise da confiabilidade do instrumento a partir das etapas descritas a seguir. Foi realizada a reversão dos escores das questões "ET1; ET2; ET3; ET4; ET5 e ET6", com a finalidade de deixar todas as variáveis com escores no mesmo sentido, pois Pestana e Gageiro (2003) afirmam que as variáveis com escores positivos e negativos no mesmo fator geram correlações negativas que violam o modelo de consistência interna e inviabilizam a utilização do coeficiente de Alfa de Cronbach. Posteriormente, foi realizada a análise do Alfa de Cronbach, em que Hair et al. (2009) afirmam ser o melhor coeficiente mensuração da confiabilidade. 
A escala MBI-GS apresentou um Alfa de Cronbach de 0,87, que Costa (2011) afirma ser uma confiabilidade ótima. Na população pesquisada foi verificada a existência do Burnout em níveis moderados, tanto para a escala quanto para as suas dimensões, exceto que a dimensão eficácia no trabalho que apresentou um índice elevado, conforme proposições de Mclaurine (2008), apresentados na Tabela 3.

Tabela 3: Médias de Burnout dos servidores da instituição

\begin{tabular}{lcc} 
& Média & Desvio padrão \\
\hline Burnout $^{2}$ & 1,52 & 0,88 \\
\hline Exaustão & 2,50 & 1,44 \\
\hline Cinismo & 1,05 & 1,19 \\
\hline Eficácia no trabalho & 5,00 & 0,98 \\
\hline
\end{tabular}

Fonte: dados da pesquisa.

Para avaliar a existência de diferenças em média entre o grupo na mensuração de Burnout aplicou-se o teste t-student que para HAIR et al. (2009) serve para avaliar se a diferença estatística entre duas amostra é significativa. Previamente utilizou-se o teste F para a avaliação da hipótese nula de igualdade das variâncias (MALHOTRA, 2012). Quando, para um nível de significância menor que 5\% rejeitou-se a hipótese nula do teste $\mathrm{F}$ e aplicou-se o teste $\mathrm{T}$ heterocedástico.

A Hipótese H1: Não existe diferença entre as profissões relacionadas à área médica e as relacionadas às áreas administrativas. Foi confirmada estatisticamente (sig 0,26$)$ para Burnout, não sendo encontradas diferenças entre as categorias que trabalham no hospital.

Os resultados corroboram com Demerouti et al. (2001) o qual alegam não existir restrições referentes à ocupação, com altas exigências no trabalho e recursos limitados, resultando em altas cargas de estresse nos trabalhadores, o Burnout pode se desenvolver independentemente do tipo de ocupação.

Quando mensuradas as dimensões do Burnout, Exaustão Emocional mostrou diferenças estatisticamente significativas (sig 0,00), onde quem trabalha nas áreas médicas apresentam uma média de Exaustão Emocional maior $(2,84)$ do que aqueles que trabalham em áreas administrativas $(1,96)$, conforme tabela 4.

Tabela 4: Teste de diferenças entre Classes de Trabalho

\begin{tabular}{|c|c|c|c|c|c|c|c|}
\hline \multirow[b]{2}{*}{ Dimensões } & \multirow[b]{2}{*}{ Classes } & \multirow{2}{*}{ Média } & \multirow{2}{*}{$\begin{array}{l}\text { Desvio } \\
\text { padrão }\end{array}$} & \multicolumn{2}{|c|}{ Teste F } & \multicolumn{2}{|c|}{ Teste $T$} \\
\hline & & & & Valor & Sig. & Valor & Sig. \\
\hline \multirow{2}{*}{ Burnout } & Médica & 1,59 & 0,86 & \multirow{2}{*}{0,03} & \multirow{2}{*}{0,86} & \multirow{2}{*}{1,14} & \multirow{2}{*}{0,26} \\
\hline & Administrativa & 1,42 & 0,93 & & & & \\
\hline \multirow{2}{*}{ Exaustão } & Médica & 2,84 & 1,45 & \multirow{2}{*}{1,94} & \multirow{2}{*}{0,17} & \multirow{2}{*}{3,94} & \multirow{2}{*}{0,00} \\
\hline & Administrativa & 1,96 & 1,28 & & & & \\
\hline \multirow{2}{*}{ Cinismo } & Médica & 1,11 & 1,07 & \multirow{2}{*}{2,39} & \multirow{2}{*}{0,12} & \multirow{2}{*}{0,73} & \multirow{2}{*}{0,47} \\
\hline & Administrativa & 0,97 & 1,35 & & & & \\
\hline \multirow{2}{*}{ Eficácia no trabalho } & Médica & 0,89 & 0,85 & \multirow{2}{*}{9,04} & \multirow{2}{*}{0,00} & \multirow{2}{*}{$-1,77$} & \multirow{2}{*}{0,08} \\
\hline & Administrativa & 1,19 & 1,14 & & & & \\
\hline
\end{tabular}

\footnotetext{
${ }^{2}$ Para cálculo do Burnout o fator Eficácia no Trabalho teve seus escores invertidos.
} 


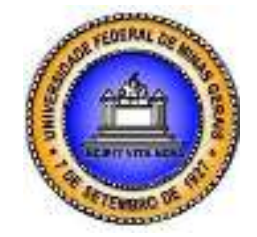

\section{cepead}

Fonte: dados da pesquisa.

Com relação à Hipótese H2: Mulheres apresentam índices maiores de Burnout que os homens. Com $95 \%$ de confiabilidade foi confirmada (sig 0,01), pois as mulheres apresentaram uma média maior $(1,65)$ do que os homens $(1,23)$ para o Burnout, essa diferença é proveniente da dimensão exaustão emocional (sig 0,00 ) onde as mulheres apresentaram uma média de 2,75 versus 1,89 dos homens, nas demais dimensões não foi comprovado existir diferenças estatisticamente significativas, conforme é apresentado na tabela 5.

Tabela 5: Teste de diferenças entre Gênero

\begin{tabular}{|c|c|c|c|c|c|c|c|}
\hline \multirow{2}{*}{ Dimensões } & \multirow{2}{*}{ Gênero } & \multirow{2}{*}{ Média } & \multirow{2}{*}{$\begin{array}{l}\text { Desvio } \\
\text { padrão }\end{array}$} & \multicolumn{2}{|c|}{ Teste $F$} & \multicolumn{2}{|c|}{ Teste $T$} \\
\hline & & & & Valor & Sig. & Valor & Sig. \\
\hline \multirow{2}{*}{ Burnout } & Masculino & 1,23 & 0,89 & \multirow{2}{*}{0,04} & \multirow{2}{*}{0,84} & \multirow{2}{*}{$-2,84$} & \multirow{2}{*}{0,01} \\
\hline & Feminino & 1,65 & 0,85 & & & & \\
\hline \multirow{2}{*}{ Exaustão } & Masculino & 1,89 & 1,39 & \multirow{2}{*}{0,11} & \multirow{2}{*}{0,74} & \multirow{2}{*}{$-3,62$} & \multirow{2}{*}{0,00} \\
\hline & Feminino & 2,75 & 1,39 & & & & \\
\hline \multirow{2}{*}{ Cinismo } & Masculino & 0,86 & 1,28 & \multirow{2}{*}{0,09} & \multirow{2}{*}{0,76} & \multirow{2}{*}{$-1,36$} & \multirow{2}{*}{0,18} \\
\hline & Feminino & 1,13 & 1,14 & & & & \\
\hline \multirow{2}{*}{ Eficácia } & Masculino & 0,85 & 0,93 & \multirow{2}{*}{1,97} & \multirow{2}{*}{0,16} & \multirow{2}{*}{$-1,37$} & \multirow{2}{*}{0,17} \\
\hline & Feminino & 1,07 & 0,99 & & & & \\
\hline
\end{tabular}

Fonte: dados da pesquisa.

Os achados corroboram com Dahlin, Fjell e Runeson (2010) que sustentam que as mulheres são mais propensas a apresentarem maiores níveis de Burnout do que os homens, muitas vezes provenientes do acumulo de tarefas no trabalho e fora dele.

Para o teste das hipóteses H3 e H4 foram realizados o teste ANOVA, que Malhotra (2012) afirma ser o teste estatístico para o estudo entre médias de duas ou mais populações. Referente à Hipótese H3: A síndrome de Burnout aumenta proporcionalmente com a idade. Com 95\% de confiabilidade não foi confirmada, pois não foi encontrada diferença estatisticamente significativa entre as médias conforme tabela 6.

Tabela 6: Teste de diferenças entre Faixas Etárias

\begin{tabular}{|c|c|c|c|c|c|c|}
\hline \multirow{2}{*}{ Dimensões } & \multirow{2}{*}{ Faixas } & \multirow{2}{*}{$\mathbf{N}$} & \multirow{2}{*}{ Média } & \multirow{2}{*}{$\begin{array}{l}\text { Desvio } \\
\text { padrão }\end{array}$} & \multicolumn{2}{|c|}{ Teste F } \\
\hline & & & & & $\mathbf{F}$ & Sig. \\
\hline \multirow{5}{*}{ Burnout } & De 23 a 35 & 27 & 1,68 & 0,92 & \multirow{5}{*}{1,12} & \multirow{5}{*}{0,33} \\
\hline & De 36 a 48 & 59 & 1,59 & 0,89 & & \\
\hline & Acima de 48 & 68 & 1,41 & 0,86 & & \\
\hline & De 36 a 48 & 63 & 0,99 & 0,93 & & \\
\hline & Acima de 48 & 72 & 0,93 & 0,98 & & \\
\hline
\end{tabular}

Fonte: dados da pesquisa.

Os resultados contrapõem os achados de Ahola et al. (2006) que afirma Burnout tem sido observado com mais frequência entre os trabalhadores jovens do que entre aqueles com idade superior a 30 ou 40 anos, não sendo encontrada diferença no presente estudo. 

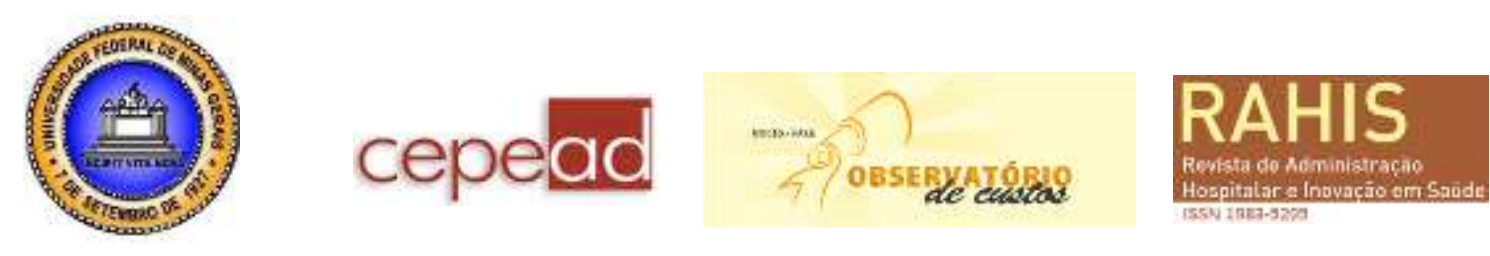

No teste da última Hipótese H4: Quanto maior o tempo de serviço na organização, maior o nível de Burnout apresentado. Não foi confirmada, com 95\% de confiabilidade, não foi encontrada diferença estatisticamente significativa (sig 0,22) entre as médias, conforme apresenta tabela 7.

Tabela 7: Teste de diferenças entre Tempo na Instituição

\begin{tabular}{|c|c|c|c|c|c|c|}
\hline \multirow{2}{*}{\multicolumn{2}{|c|}{ Tempo de Serviço }} & \multirow{2}{*}{$\mathbf{N}$} & \multirow{2}{*}{ Média } & \multirow{2}{*}{$\begin{array}{l}\text { Desvio } \\
\text { padrão }\end{array}$} & \multicolumn{2}{|c|}{ Teste F } \\
\hline & & & & & $\mathbf{F}$ & Sig. \\
\hline \multirow{4}{*}{ Burnout } & Até 6 Anos & 32 & 1,38 & 0,92 & \multirow{4}{*}{0,52} & \multirow{4}{*}{0,67} \\
\hline & De 7 a 12 Anos & 31 & 1,65 & 0,92 & & \\
\hline & De 13 a 20 anos & 39 & 1,54 & 0,90 & & \\
\hline & $\begin{array}{l}\text { Acima de } 20 \\
\text { anos }\end{array}$ & 51 & 1,50 & 0,82 & & \\
\hline
\end{tabular}

Fonte: dados da pesquisa.

Ahola et al. (2006) afirmam que o Burnout parece ocorrer muito cedo na carreira profissional, explicado por um choque de realidade, a socialização profissional bem-sucedida, ou um efeito do trabalhador saudável, o que não foi presenciado no estudo.

\section{CONSIDERAÇÕES FINAIS}

Em um ambiente de tratamento médico, onde as ações têm reflexos na saúde dos pacientes, exacerba a necessidade de atenção com o desenvolvimento da síndrome de Burnout em seus trabalhadores, com esta finalidade foi realizada a pesquisa com os servidores do hospital público, resultando em 173 respondentes com um perfil formado por $69,4 \%$ do sexo feminino e $30,6 \%$ do sexo masculino, com 44,8\% apresentando idade superior a 48 anos, demonstrando experiência na função, pois $33,3 \%$ dos pesquisados afirmou ter mais de 20 anos na função.

A amostra foi composta ainda por diversos cargos, sendo $61 \%$ ligados à área médica e $39 \%$ ligadas a administrativa, o que validou a utilização da escala MBI-GS, quanto à escala, esta apresentou boa fatorabilidade com KMO 0,837, e após procedimentos de limpeza da escala (exclusão da variável EE6) apresentou uma estrutura de três fatores conforme a literatura, sendo eles, Exaustão Emocional, Cinismo e Eficácia no Trabalho, a confiabilidade da MBI-GS foi ótima, com um Alfa de Cronbach de 0,87.

Na mensuração dos níveis de Burnout dos servidores do Hospital Público, surge um alerta, pois os mesmos apresentam níveis moderados de Burnout $(1,52)$ e para os fatores Exaustão Emocional $(2,50)$ e Cinismo $(1,05)$, somente o fator Eficácia no Trabalho apresenta um nível alto. Lindblom et al. (2006) afirmam que o Burnout está associado a outros tipos de distúrbios psicológicos, como depressão, ansiedade e insônia, o que em um ambiente de exige cuidados com pacientes deve despertar maiores atenções por parte da direção da organização a fim de minimizar os sintomas do Burnout nos trabalhadores.

Quanto aos testes de hipóteses foi confirmado que a síndrome de Burnout se desenvolve independente de qual cargo ou área a pessoa trabalha, também foi reforçado o que a literatura sobre o assunto propõe que as mulheres estão predispostas a apresentarem níveis mais altos de Burnout que os homens. Quanto à associação com a experiência dos trabalhadores em relação 

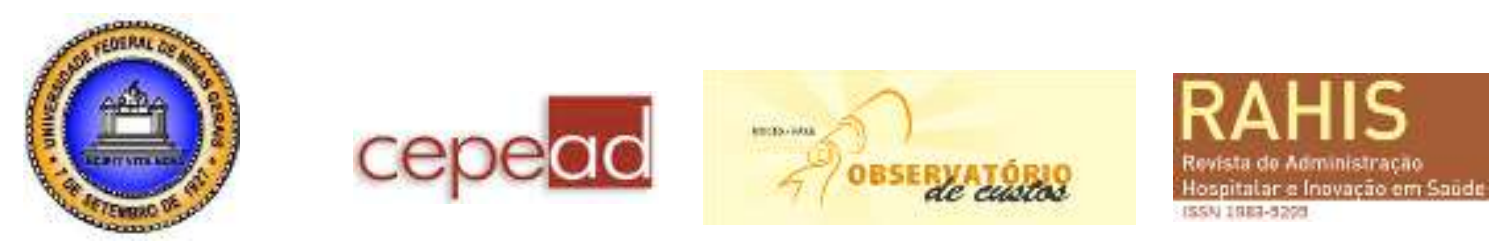

a sua idade e desempenho da função, não foi encontrada relação com o desenvolvimento do Burnout.

O presente estudo colabora com o estudo da síndrome de Burnout, através da aplicação da escala MBI-GS em diversos cargos de um hospital público, a escala em questão mostrou-se adaptada a população brasileira, apresentando boa fatorabilidade, sem necessidades de grandes exclusões de variáveis e uma ótima confiabilidade.

Como limitações do estudo foram encontrados poucos estudos que utilizaram a mesma escala, não sendo possível realizar a comparação dos índices encontrados, também foi uma limitação a resistência dos trabalhadores do hospital em responder os questionários. Ficando como sugestão para futuras pesquisas a replicação da escala em setores diferentes e em uma população maior.

\section{REFERENCIAS}

AHOLA, K. et al. Burnout in the general population - Results from the Finnish Health 2000 Study. Social Psychiatry and Psychiatric Epidemiology, v. 41, n. 1, p. 11-17, Jan 2006. ISSN 0933-7954. Disponível em: < <Go to ISI>://WOS:000235450500002 >.

BACKES, D. S.; LUNARDI FILHO, W. D.; LUNARDI, V. L. O processo de humanização do ambiente hospitalar centrado no trabalhador. Revista da Escola de Enfermagem da USP, v. 40, p. 221-227, 2006. ISSN 0080-6234. Disponível em: < http://www.scielo.br/scielo.php?script=sci_arttext\&pid=S008062342006000200010\&nrm=iso >.

BAKKER, A. B.; DEMEROUTI, E.; SCHAUFELI, W. B. Validation of the Maslach Burnout Inventory - General survey: An internet study. Anxiety Stress and Coping, v. 15, n. 3, p. 245260, Sep 2002. ISSN 1061-5806. Disponível em: <<Go to ISI >://WOS:000178103900003 >.

BAKKER, A. B.; VAN EMMERIK, H.; EUWEMA, M. C. Crossover of burnout and engagement in work teams. Work and Occupations, v. 33, n. 4, p. 464-489, Nov 2006. ISSN 0730-8884. Disponível em: <<Go to ISI>://WOS:000241331900009>.

COSTA, F. J. D. Mensuração e Desenvolvimento de Escalas: Aplicações em Administração. $1^{\text {a }}$. Rio de Janeiro: Editora Ciencia Moderna Ltda, Rio de Janeiro, 2011. ISBN 9788539901449.

DAHLIN, M.; FJELL, J.; RUNESON, B. Factors at medical school and work related to exhaustion among physicians in their first postgraduate year. Nordic Journal of Psychiatry, v. 64, n. 6, p. 402-408, Dec 2010. ISSN 0803-9488. Disponível em: < <Go to ISI $>: / /$ WOS:000284165700008 >.

DEMEROUTI, E. et al. The job demands-resources model of burnout. Journal of Applied Psychology, v. 86, n. 3, p. 499-512, Jun 2001. ISSN 0021-9010. Disponível em: < <Go to ISI > :/WOS:000170878300012 >.

ETZION, D. Moderating Effect of Social Support on The Stress Burnout Relationship. Journal of Applied Psychology, v. 69, n. 4, p. 615-622, 1984 1984. ISSN 0021-9010. Disponível em: $<<$ Go to ISI $>$ ://WOS:A1984TU11100010 >. 

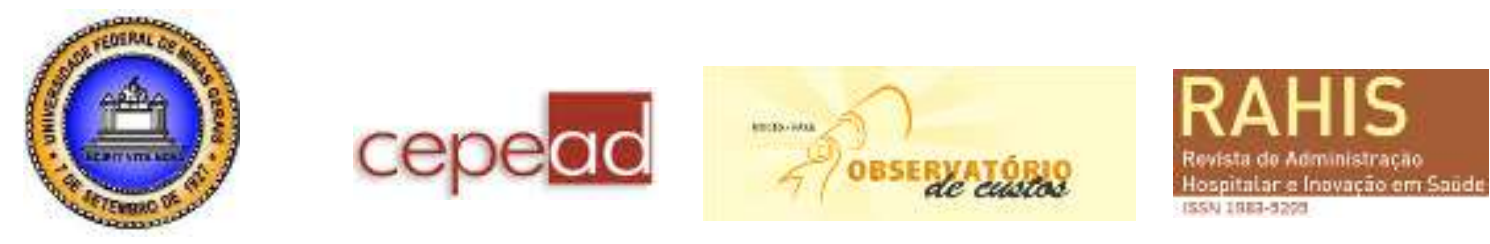

FERREIRA, R. E. D. D. S. Dissertação de Mestrado. A organização do trabalho na Unidade de Doenças Infecto-contagiosas e a ocorrência de Burnout nos trabalhadores de Enfermagem. bdtd.uerj.br, 2011. Disponível em: < http://www.bdtd.uerj.br/tde_arquivos/20/TDE-2011-1018T131135Z-1898/Publico/dissertacao_final_rita_elzi_dias_de_seixas_ferreira.pdf > .

HAIR, J. F. et al. Análise multivariada de dados. Bookman. Porto Alegre v. 6. Ed., 2009.

HALBESLEBEN, J. R. B.; BUCKLEY, M. R. Burnout in organizational life. Journal of Management, v. 30, n. 6, p. 859-879, 2004. ISSN 0149-2063. Disponível em: < <Go to ISI $>$ ///WOS:000224007800006 >.

JACKSON, S. E.; SCHWAB, R. L.; SCHULER, R. S. TOWARD AN UNDERSTANDING OF THE BURNOUT PHENOMENON. Journal of Applied Psychology, v. 71, n. 4, p. 630640, Nov 1986. ISSN 0021-9010. Disponível em: <<Go to ISI>://WOS:A1986E941300013>.

LEE, R. T.; ASHFORTH, B. E. A meta-analytic examination of the correlates of the three dimensions of job burnout. Journal of Applied Psychology, v. 81, n. 2, p. 123-133, Apr 1996. ISSN 0021-9010. Disponível em: < <Go to ISI>://WOS:A1996UF81900001 >.

LINDBLOM, K. M. et al. Burnout in the working population: Relations to psychosocial work factors. International Journal of Behavioral Medicine, v. 13, n. 1, p. 51-59, 2006. ISSN 1070-5503. Disponível em: < <Go to ISI >://WOS:000235943900007 >.

MAlHOTRA, N. K. Pesquisa de Marketing: uma orientação aplicada. Porto Alegre: Bookman, 2012. ISBN 9788577809752.

MASLACH, C. Job burnout: New directions in research and intervention. Current Directions in Psychological Science, v. 12, n. 5, p. 189-192, Oct 2003. ISSN 0963-7214. Disponível em: $<\langle$ Go to ISI $>$ ://WOS:000185645800009 >.

MASLACH, C.; GOLDBERG, J. Prevention of burnout: New perspectives. Applied \& Preventive Psychology, v. 7, n. 1, p. 63-74, Win 1998. ISSN 0962-1849. Disponível em: < $<$ Go to ISI >://WOS:000071497000004 >.

MASLACH, C.; SCHAUFELI, W. B.; LEITER, M. P. Job burnout. Annual Review of Psychology, v. 52, p. 397-422, 2001. ISSN 0066-4308. Disponível em: < <Go to ISI > :/WOS:000167463100017 >.

MCLAURINE, W. D. A Correlational Study of Job Burnout and Organizational Commitment Among Correctional Officers. School of Psychology: Capella University 2008.

MONTERO-MARIN, J. et al. Burnout syndrome among dental students: a short version of the "Burnout Clinical Subtype Questionnaire" adapted for students (BCSQ-12-SS). BMC Medical Education, v. 11, Dec 12 2011. ISSN 1472-6920. Disponível em: < <Go to ISI $>$ ://WOS:000300156100001 >.

PESTANA, M. H.; GAGEIRO, J. N. Análise de dados para Ciências Sociais: a complementaridade do SPSS. Sílabo, 2003. ISBN 9789726184980. 
PRUESSNER, J. C.; HELLHAMMER, D. H.; KIRSCHBAUM, C. Burnout, perceived stress, and cortisol responses to awakening. Psychosomatic Medicine, v. 61, n. 2, p. 197-204, MarApr 1999. ISSN 0033-3174. Disponível em: < <Go to ISI>://WOS:000079301000012 >.

SCHAUFELI, W. B.; GREENGLASS, E. R. Introduction to special issue on burnout and health. Psychology \& Health, v. 16, n. 5, p. 501-510, 2001. ISSN 0887-0446. Disponível em: $<<$ Go to ISI $>$ ://WOS:000171388900001 >. 